\title{
Number magnitude to finger mapping is disembodied and topological
}

\author{
Myrthe A. Plaisier · Jeroen B. J. Smeets
}

Received: 27 August 2010/Accepted: 17 January 2011/Published online: 6 February 2011

(C) The Author(s) 2011. This article is published with open access at Springerlink.com

\begin{abstract}
It has been shown that humans associate fingers with numbers because finger counting strategies interact with numerical judgements. At the same time, there is evidence that there is a relation between number magnitude and space as small to large numbers seem to be represented from left to right. In the present study, we investigated whether number magnitude to finger mapping is embodied (related to the order of fingers on the hand) or disembodied (spatial). We let healthy human volunteers name random numbers between 1 and 30, while simultaneously tapping a random finger. Either the hands were placed directly next to each other, $30 \mathrm{~cm}$ apart, or the hands were crossed such that the left hand was on the right side of the body mid-line. The results show that naming a smaller number than the previous one was associated with tapping a finger to the left of the previously tapped finger. This shows that there is a spatial (disembodied) mapping between number magnitude and fingers. Furthermore, we show that this mapping is topological rather than metrically scaled.
\end{abstract}

Keywords Number processing - Spatial representation . Embodied representation - Finger position

\section{Introduction}

An increasing number of studies suggest that finger representation and numerical cognition are closely related. Using brain images techniques, it has been shown that

M. A. Plaisier $(\bowtie) \cdot$ J. B. J. Smeets

Faculty of Human Movement Sciences, VU University,

Van der Boechorststraat 9, 1081 BT Amsterdam,

The Netherlands

e-mail: m.plaisier@fbw.vu.nl brain areas involved in number processing overlap with those involved in finger movements (Pesenti et al. 2000). In patients with Gerstmann syndrome, numerical deficits are sometimes accompanied by finger agnosia (Martory et al. 2003; Mayer et al. 1999). Furthermore, it has been shown that there is cortical spinal excitability specifically for the hand muscles during numerical judgements (Andres et al. 2007; Sato et al. 2007). This association between finger representation and number processing has been explained as reminiscent from finger counting strategies used during childhood (Butterworth 1999). Whether finger counting strategies indeed cause this overlap between number processing and finger movement control areas in the brain is still open for debate. It is, however, clear that finger counting strategies interact with numerical judgements (Badets et al. 2010; Fischer 2008; Di Luca et al. 2006). This interaction suggests that there is an association between specific numbers and specific fingers. Such a relationship is referred to as an embodied association.

Besides a relation between the fingers and number processing, there is also a vast amount of evidence for associations between space and number processing (see Hubbard et al. (2005) for a review). For instance, in bisection of digit strings (e.g. 11111 or 77777), a bias to the left or right is found depending on whether large or small digits are shown, respectively (Fischer 2001). Such a bisection bias has also been shown cross-modally between a visually presented arabic digit and haptic bisection of a rod (Cattaneo et al. 2010). Perhaps the most frequently replicated demonstration of interactions between space and number magnitude is the SNARC effect (Dehaene et al. 1993). When healthy human volunteers are asked to judge the parity of a presented digit by pressing a button, it has been found that responses are faster with the hand on the left side of the body mid-line for small numbers and with 
the hand on the right side of the body mid-line for large numbers. This effect persists when the hands are crossed, indicating that the faster performance for small and large numbers is related to the left and right side of space. This spatial association has led to the idea that humans represent numbers from left to right on a logarithmically scaled internal number line (Dehaene 2003). The SNARC effect demonstrates an association between numbers and the actual positions of the fingers in space. We will refer to this mapping as disembodied. Such a mapping between numbers and the position of the fingers in space contrasts with the previously described embodied mapping between specific numbers and specific fingers. Based on these results, one could hypothesise that embodied mapping is specific to situations where the numbers one to ten can each be mapped onto one specific finger, whereas numerical magnitude, or any other type of magnitude, is mapped in a disembodied way.

In a situation where it was possible to map a specific number to a specific finger, dominance of a disembodied mapping over an embodied mapping has been demonstrated. In an attention priming study by Brozzoli et al. (2008), a tactile stimulus was presented to the thumb or little finger after a digit was shown. Subjects responded faster to a stimulus that was presented to a finger on the left side of the hand after seeing a small digit than after seeing a large digit $(<5)$. This effect was independent of whether the palm of the hand was facing upward or downward, showing that disembodied mapping was dominant over embodied mapping. Note that this finding is not in conflict with our hypothesis. Although it was possible in this situation to map specific numbers to specific fingers, also mapping of numerical magnitude probably played a role here.

A recent study investigated the relation between numbers and eye movements (Loetscher et al. 2010). In this study, volunteers were asked to name a 'random' number every second while their eye movements were recorded. The results show that naming a larger number than the previous one correlated with an eye movement to the right. Similarly, naming a smaller number was associated with an eye movement towards the left. Larger numerical differences were on average accompanied by larger shifts in eye position. This shows an association between space and numbers. Clearly, in this case, there was no possibility of an embodied mapping as there is between fingers and numbers. Therefore, one could conclude from this result that mapping of magnitude is disembodied. If indeed the mapping of magnitude is primary disembodied, then this should also hold for magnitude to finger mapping. Therefore, we expect that there is a relationship between subsequently tapped fingers and subsequently named numbers, similar to the relation between a shift in eye position and the difference in numerical magnitude.
In contrast to studies investigating the effects of finger counting strategies, our experiment was set up such that it was impossible to associate one specific finger with one specific number. This ensures that we investigate the mapping between relative number magnitude and the positions of the fingers. Using this paradigm, we can determine whether large numerical differences are associated with tapping a finger located relatively far from the previously tapped finger. Note that in contrast to eye positions, the fingers occupy discrete positions in space and the fingers are attached to the hands in a certain order. If the mapping between tapped fingers and number magnitude is based on the order of the fingers on the hand, we will consider this an 'embodied' mapping; while if it is based on the positions of the fingers in space, we will consider the mapping 'disembodied'. In the latter case, we expect our results to be independent of whether the hands are crossed such that the left hand is on the right side of the body mid-line.

If the magnitude to finger mapping is found to be disembodied in our task, we will investigate whether this mapping is metric or topological. A topological distance refers to the number of fingers between one finger and another finger, while the metric distance is expressed in metric units such as centimetres. In other words, if the mapping is topological, the middle fingers of each hand are considered spaced equally far apart when the hands are placed adjacent or, for instance, $30 \mathrm{~cm}$ apart, because the number of fingers in between the two middle fingers remains the same. If the mapping is metric, spacing the hands further apart should yield different results compared with placing the hands directly adjacent to each other, whereas the results will remain the same if mapping is topological.

\section{Methods}

\section{Participants}

Ten employees (two men, one left-handed, aged 22-32 years) of the Faculty of Human Movement Sciences of VU University participated in the experiment. All subjects were naive as to the purpose of the experiment. The experiment was part of a research programme approved by the ethics committee of the faculty of Human Movement Sciences.

\section{Set-up}

The set-up consisted of contact sensors that were placed under the subject's fingers. A box was placed over the setup such that the subjects could not see their hands. The 
contact sensors were sampled at $50 \mathrm{~Hz}$. Named numbers were recorded using a microphone.

\section{Experimental design}

Subjects were seated with their fingers slightly above the sensors. They were instructed to name a number between 1 and 30 'as random as possible', paced by a beep at $1 \mathrm{~Hz}$. We chose the maximum value to be larger than 10 to prevent a possible fixed mapping between a number and a digit; otherwise, the value of 30 was chosen arbitrarily. Subjects were asked to try to adhere to the maximum number of 30 but were told not to worry when they accidentally named a larger number and to just keep on going. It was more important for the subject to keep the pace than to stay exactly within the numerosity range. There were no other restrictions on the named numbers for the subjects, and they were allowed to name the same number consecutively. With every named number, they randomly tapped one finger of either hand, excluding the thumb and little finger. All subjects performed three conditions, and the order was roughly counterbalanced over subjects. In each condition, the subjects named 300 numbers in consecutive blocks of 100 numbers with breaks of a few minutes in between. Before each condition started, a practice block of 100 trials was performed to allow subjects to become comfortable with the task and get acquainted with the pace of the beeps. In the 'Close' condition the hands were placed such that the distance between subsequent fingers was always $2.5 \mathrm{~cm}$ (Fig. 1). In the 'Wide' condition the hands were placed $30 \mathrm{~cm}$ apart (distance between the left and right index fingers in the 'Wide' condition and between the right and left ring fingers in the 'Crossed' condition). Finally, in the 'Crossed' condition the hands were also placed $30 \mathrm{~cm}$ apart, but the left hand was on the right side of the body mid-line and vice versa. None of the subjects reported accidentally tapping the thumb or little fingers.

After the experiment, subjects were asked to count from one to ten on their fingers. All subjects started with the thumb of either the left or right hand, passed all fingers of that hand and continued with the thumb of the other hand. We will come back to this in the discussion.

\section{Analysis}

For the analysis, we numbered the digits $1-6$ from left to right. Note that this means that in the 'Crossed' condition, the right index finger was labelled 1 in the analysis, while in the other two conditions the left ring finger was labelled 1. The differences between the subsequently named numbers were calculated as well as the differences between the subsequently tapped fingers. Because subjects quite frequently responded 31 or 32 , these numbers were included
Close

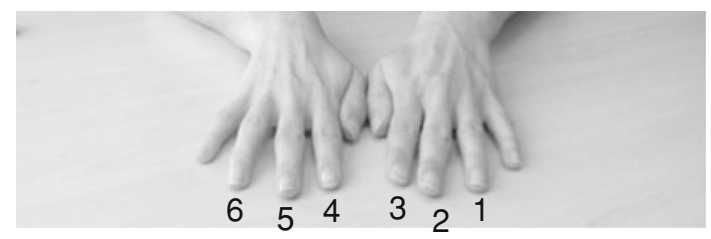

Wide
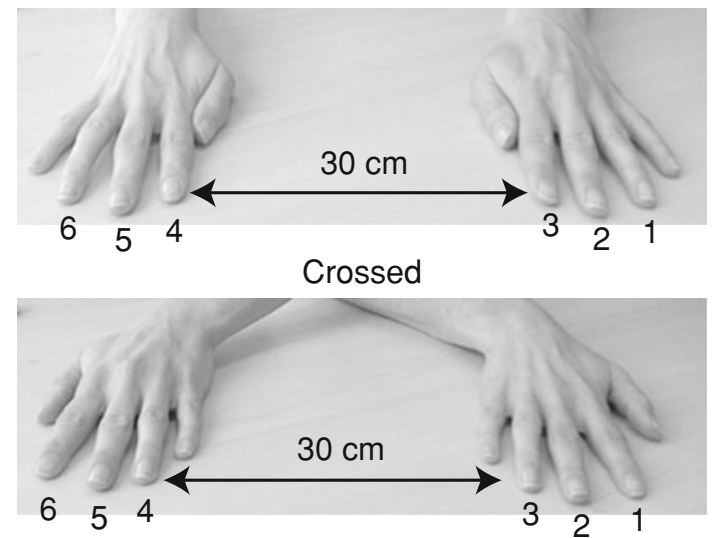

Fig. 1 The position of the hand in the three conditions. In the 'Close' condition', the hands were placed directly next to each other, while in the 'Wide' and 'Crossed' conditions, the hands were placed $30 \mathrm{~cm}$ apart. In the 'Crossed' conditions, the hands were placed such that the left hand was on the right side of the body mid-line and vice versa. The numbers indicate the numbering of the fingers as used in our analysis

in the analysis. Trials in which a larger number was named (the largest number named was 52) were discarded as missed trials $(0.2 \%$ of all trials). Also trials in which subjects failed to tap a finger or to name a number before the next beep were discarded from the analysis.

Differences between subsequent trials were taken until there was a missing trial. After the missing trial, taking differences between subsequent trials was continued. This means that no differences between trials before and after a missing trial were taken and only differences between consecutive trials were analysed. The data were then collapsed over subjects, and for each finger position difference, the average difference between the named numbers was calculated. To distinguish between a metric and a topological representation, the difference between subsequently named numbers was analysed as a function of both the ordinal distance and the metric distance between subsequently tapped fingers. Therefore, linear regression weighted to the standard error was performed on the data from each condition separately.

\section{Results}

Figure $2 \mathrm{a}$ shows the percentage of trials in which each of the six included fingers was tapped averaged for each of the 
Fig. 2 a Bar charts showing for all of the conditions the percentage of trials in which percentage of switches between one finger to another finger for all possible finger combinations. The squares on the diagonal fingers twice in a row. $\mathbf{c}$ The bar chart shows the percentage of trials in which a certain number was named averaged over all conditions. d The matrix in which two numbers were named subsequently for all possible number combinations averaged over all conditions. The squares on the diagonal indicate naming the same number twice in a row each finger was tapped. $\mathbf{b}$ The indicate tapping the same indicates the percentage of trials
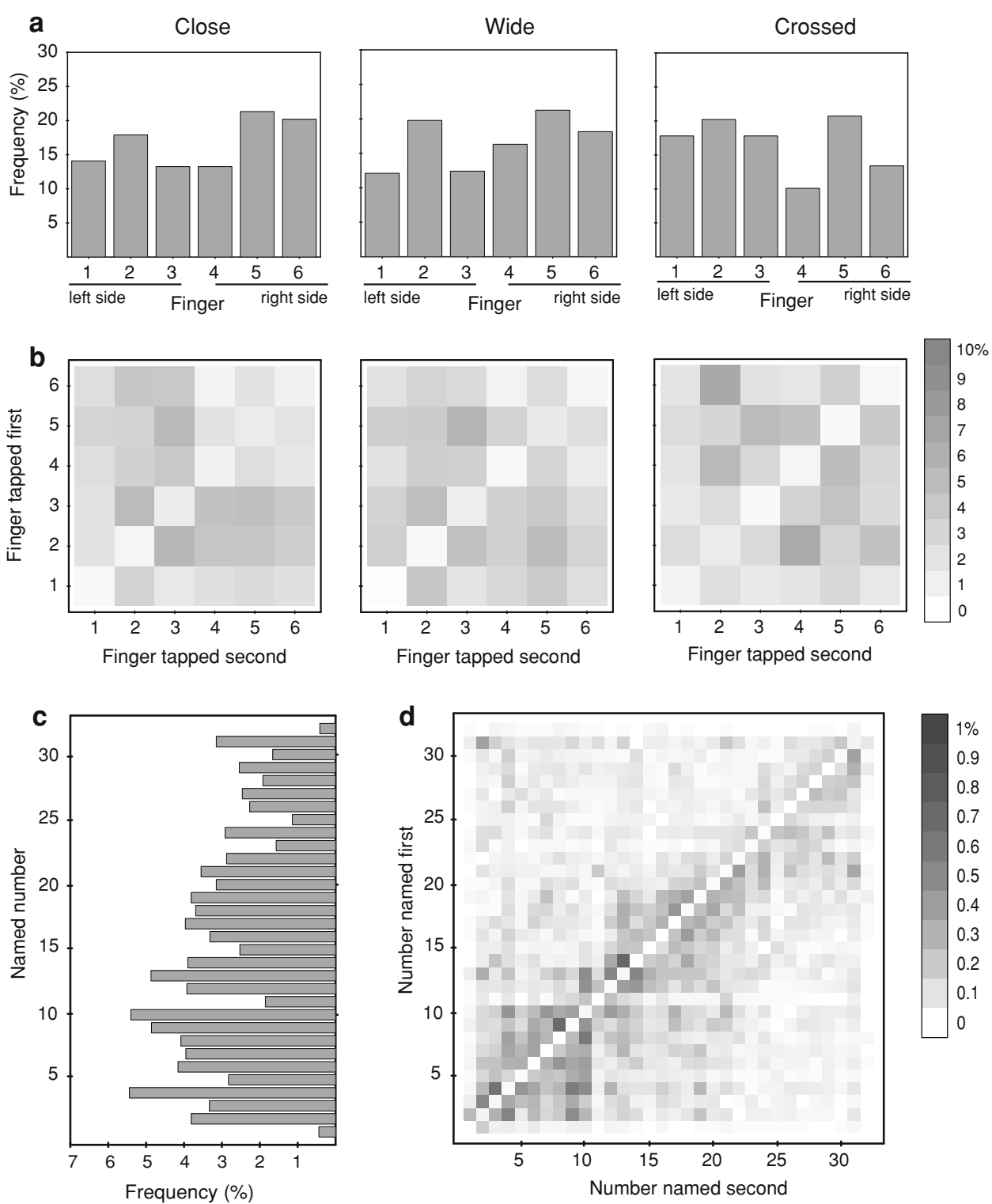

conditions. It can be seen that all fingers were used with a slight preference for the middle finger in all conditions. The percentage of trials in which two fingers were subsequently tapped is shown in Fig. 2b. Since all squares in the matrices have a grey value, it can be seen that all possible finger combinations occurred, but that subjects generally refrained from tapping the same finger subsequently (lightcoloured squares on the diagonal). Because these matrices are symmetrical around the diagonal, there were switches from left to right hand equally often as switches from right to left hand in each of the conditions. So, the smaller distance between the hands in the 'close' conditions did not lead to an evidently different number of switches between the two hands. Figure $2 \mathrm{c}$ shows the percentage of trials in which a certain number was named averaged over all conditions. From this figure, it is clear that all numbers in the range were named. In Fig. $2 d$, it can be seen that subjects tended to name numbers that were numerically relatively close together (dark squares near the diagonal). They did, however, not very often name the same number subsequently (light squares on the diagonal). These results show that the subjects were able to comply with the task. They were able to keep the pace, and they used all fingers and the whole numerosity range.

For each of the three conditions, a linear regression was performed on the difference between subsequently named numbers as a function of the difference between subsequently tapped fingers (Fig. 3a). Note that differences between subsequently named numbers were analysed, meaning that they ranged from -31 to +31 . Because of the positive and negative numbers averaging out to much smaller values, the axes only run from -4 to +4 . 


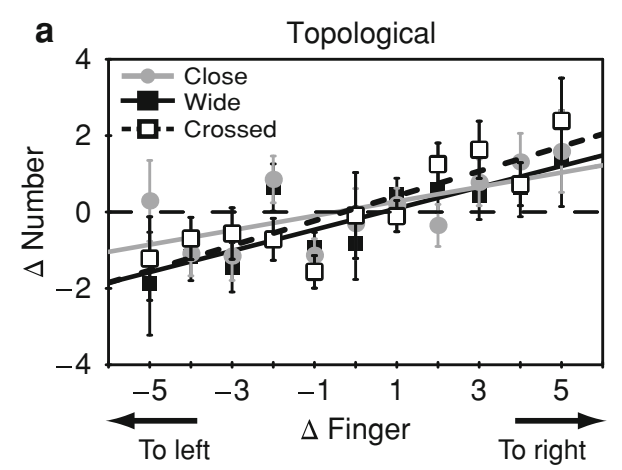

b

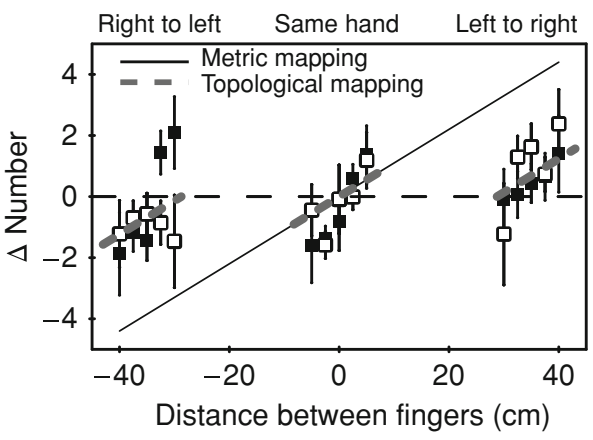

Fig. 3 a Difference between two subsequent numbers as function of the number of fingers between the subsequently tapped fingers averaged over all subjects for each of the three conditions. The lines indicate linear regression to data from each of the conditions. b 'Wide' and 'Crossed' conditions as a function of the metric distances between the fingers. Points at distances below $-30 \mathrm{~cm}$ represent switches from a finger of the hand on the right side of the body mid-line to a finger of the hand on the left side, switches from the hand on the left side of the body mid-line to the hand on right side are centred at distances larger than $30 \mathrm{~cm}$ and switches between fingers of the same hand around $0 \mathrm{~cm}$. The solid line shows the prediction for metric mapping and the dotted line for topological mapping. Error bars indicate the standard error. Although differences between subsequently named numbers ranged from -31 to 31 , the presented scale is smaller because only means and standard errors are reported here

A least-squares minimisation procedure weighted to the inverse squared standard errors for named number was used, taking into account that there were discrete values for differences in distance between the fingers. The slope values were positive (close: 0.19 , wide: 0.28 , crossed: 0.32 ) and were significantly different from zero $(p \leq 0.03)$, but not from each other $(p \geq 0.09)$. The positive slopes in all conditions, including 'Crossed', refute an embodied mapping.

For the 'Close' condition, distances between all neighbouring fingers were the same and the metric and topological representations yield the same predictions. To distinguish between both representations, we plotted the difference between subsequently named numbers as a function of the metric distance between subsequently tapped fingers for the 'Wide' and 'Crossed' conditions (Fig. 3b). The slope of magnitude difference as a function of the finger number difference is converted to the slope of the magnitude difference as a function of the metric distance between fingers. Based on the average slope over all conditions in Fig. 3a (0.26), and the distance between subsequent fingers $(2.5 \mathrm{~cm})$, we predict a metric slope of $\left(\frac{0.26}{2.5 \mathrm{~cm}}=\right) 0.11 \mathrm{~cm}^{-1}$. If number to finger mapping is metric, a single line with a slope of $0.11 \mathrm{~cm}^{-1}$ passing trough zero at $0 \mathrm{~cm}$ should describe the data (continuous line in Fig. 3b). In the case of topological mapping, we predict three linear regimes with a slope of $0.11 \mathrm{~cm}^{-1}$ in which naming a larger number is associated with tapping a finger to the right of the previous finger regardless of the metric distance (dashed lines in Fig. 3). It can be seen that the topological model predicts the data best $\left(\mathrm{R}^{2}=0.45\right.$ averaged over both conditions). The metric model even yields negative explained variance values, indicating that the model prediction was worse than the weighted average.

Based on the SNARC effect, it could be expected that subjects named a smaller number when tapping with the left hand than when tapping with the right hand. To test whether this was the case, named numbers for the hand on the right side and the hand on the left side of the body midline were averaged for each subject in each condition. A paired samples $t$ test (two-tailed) was performed on the averages from all the subjects in each condition separately. This analysis did show a tendency towards naming smaller numbers when tapping with the left hand than when tapping with the right hand, but this effect did not reach significance in any of the three conditions $(t(9)=-2.1$, $p=0.07, t(9)=-1.3, p=0.21 \quad$ and $\quad t(9)=-0.91$, $p=0.38)$.

\section{Discussion}

Our results show that subjects associated naming a smaller number with tapping a finger to the left of the previous finger and naming a larger number with tapping a finger to the right. Furthermore, this effect was unchanged when the hands were crossed. This shows that the mapping between number magnitude and the fingers was related to the position of the fingers in space, not the order of the fingers on the hands. Note that none of our subjects' finger counting habits could explain such results nor could any other finger counting habit. Because we investigated the relationship between differences between subsequently tapped fingers and differences in the numbers named, only a rare finger counting habit of starting with the little finger of one hand and continuing with the thumb of the other hand would yield a correlation from left to right from small to large numbers. This strategy would, however, yield a reversed correlation in the condition with the hands 
crossed, and our results clearly show that this was not the case. Because our results only depend on the position of the fingers in space and not the order of the fingers on the hand, we can conclude that there was a disembodied mapping from left to right for small to large numerical values.

Furthermore, when the hands were placed $30 \mathrm{~cm}$ apart, the mapping between number magnitude and finger was not scaled to metric space. A large numerical difference between two subsequently named numbers was accompanied by tapping a finger that was separated by a large number of fingers from the previously tapped finger regardless of the distance between the hands. This shows that number magnitude to finger mapping is topological, meaning that there is a spatial structure, but no metric scaling. From visuomotor tasks, it has also been suggested that the brain represents locations in a topological fashion (Thaler and Goodale 2010).

The present study does not refute any embodied association between numbers and fingers. It has been demonstrated that there are interactions between numbers and finger counting strategies (Badets et al. 2010; Fischer 2008; Di Luca et al. 2006). Such a mapping between specific numbers and specific fingers clearly is an embodied one. However, in our experiment, there was no unique association between a single finger and a single number possible because the range of numbers was larger than the number of fingers. In this case, there is a magnitude mapping, not a mapping between a specific number and a specific number. Our results clearly show that magnitude to finger mapping is related to the position of the fingers in space and not the order of the fingers on the hand. Increasing numerical values were associated with the fingers through a topological spatial mapping from left to right. Therefore, we can conclude that magnitude to finger mapping is disembodied through a topological spatial mapping from left to right.

Acknowledgments This research was supported by a VICI grant (MaGW 453-08-004) from the Netherlands Organisation for Scientific Research (NWO).

Open Access This article is distributed under the terms of the Creative Commons Attribution Noncommercial License which permits any noncommercial use, distribution, and reproduction in any medium, provided the original author(s) and source are credited.

\section{References}

Andres M, Seron X, Olivier E (2007) Contribution of hand motor circuits to counting. J Cogn Neurosci 19:563-576

Badets A, Pesenti M, Olivier E (2010) Response-effect compatibility of finger-numeral configurations in arithmetical context. Q J Exp Psychol 63(1):16-22

Brozzoli C, Ishihara M, Goebel SM, Salemme R, Rossetti Y, Farne A (2008) Touch perception reveals the dominance of spatial over digital representation of numbers. Proc Natl Acad Sci USA 105(14):5644-5648

Butterworth B (1999) Perspectives: neuroscience-a head for figures. Sci Agric 284(5416):928-929

Cattaneo Z, Fantino M, Tinti C, Silvanto J, Vecchi T (2010) Crossmodal interaction between the mental number line and peripersonal haptic space representation in sighted and blind individuals. Atten Percept Psychophys 72(4):885-890

Dehaene S (2003) The neural basis of the Weber-Fechner law: a logarithmic mental number. Trends Cogn Sci 7(4):145-147

Dehaene S, Bossini S, Giraux P (1993) The mental representation of parity and number magnitude. J Exp Psychol Gen 122:371-396

Di Luca S, Grana A, Semenza C, Seron X, Pesenti M (2006) Fingerdigit compatibility in Arabic numeral processing. Q J Exp Psychol 59(9):1648-1663

Fischer MH (2001) Number processing induces spatial performance biases. Neurology 57(5):822-826

Fischer MH (2008) Finger counting habits modulate spatial-numerical associations. Cortex 44:386-392

Hubbard EM, Piazza M, Pinel P, Dehaene S (2005) Interactions between number and space in parietal cortex. Nat Rev Neurosci 6:435-448

Loetscher T, Bockisch CJ, Nicholls ME, Brugger P (2010) Eye position predicts what number you have in mind. Curr Biol 20:R264-R265

Martory M, Mayer E, Pegna A, Annoni J, Landis T, Khateb A (2003) Pure global acalculia following a left subangular lesion. Neurocase 9(4):319-328

Mayer E, Martory M, Pegna A, Landis T, Delavelle J, Annoni J (1999) A pure case of Gerstmann syndrome with a subangular lesion. Brain Behav Evol 122(Part 6):1107-1120

Pesenti M, Thioux M, Seron X, De Volder A (2000) Neuroanatomical substrates of arabic number processing, numerical comparison, and simple addition: A PET study. J Cogn Neurosci 12:461-479

Sato M, Cattaneo L, Rizzolatti G, Gaffese V (2007) Numbers within our hands: Modulation of corticospinal excitability of hand muscles during numerical judgment. J Cogn Neurosci 19(4):684-693

Thaler L, Goodale MA (2010) Beyond distance and direction: The brain represents target locations non-metrically. J Vis 10(3):1-27 